\title{
Externally Liquid-Filled Photonic Crystal Fibers with High Output Intensity and Low Confinement Loss
}

\author{
M. HosseinPour* AND A. ZENDEHNAM \\ Department of Physics, Faculty of Science, Arak University, P.O. Box 38156-8-8349, Arak, Iran
}

(Received April 13, 2015; in final form April 4, 2017)

In this study, a Gaussian function is used to approximate the output intensity profiles of the externally liquidfilled photonic crystal fibers with various configurations. By decreasing the diameters of the five outer rings of the proposed photonic crystal fibers, the output intensity values are shown to be considerably increased for all of the studied configurations. A very high output intensity value with very low confinement loss (roughly $0 \mathrm{~dB} / \mathrm{m}$ ) can be achieved at $\lambda=1.55 \mu \mathrm{m}$ by an appropriate design for the externally liquid-filled PCF with nanostructure.

DOI: 10.12693/APhysPolA.131.1431

PACS/topics: 42.81.-i, 42.81.Pa, 42.15.Eq

\section{Introduction}

Photonic crystal fibers (PCFs) classified according to their core, which is solid or air, are considered to be a microstructured optical fiber (MSOF) or holey fiber (HF). These fibers are not only excelled to perform very well in non-telecom or telecom wavelength range, but also they exhibit unique properties. This innovative class of fibers is fabricated by embedding a number of air channels which as usual form square [1] or hexagonal designs [2] around the core and run along the fiber.

These unique characteristics that provide the possibility of altering PCFs ability to concentrate the mode field in the core region by inducing a small change on air-hole structure, have made these fibers to be more interesting than conventional fibers. To illustrate the dependence of PCFs performance and structural parameters such as the diameter of air holes $(d)$ or the hole pitch $(\Lambda)$, recently, comprehensive designs which have proposed several distinctive amounts for these parameters, have been studied [3-5]. In the recent decades, a variety of liquids, such as index oils [6, 7], polymers [8], liquid crystals [9], and even metals [10] have been employed to infiltrate into the holes of the PCFs to form the liquid-filled PCFs. These sorts of PCFs can be utilized in optical sensing [11], switching [12], or communications [13]. Furthermore, in some previous articles, the hollow core (HC) PCFs filled with different liquids in the air core or air cladding, have been investigated [14-16].

Selectively liquid-filled PCFs with very low chromatic dispersion value by varying the air-hole diameters and liquid indices [17] as well as the high-index externally and internally liquid-filled PCFs with low confinement loss (CL) by variation in the number of air-hole layers have been reported [18]. However, as far as we know, none of previous studies have given comprehensive insight into new designs of selectively liquid-filled PCFs with various

\footnotetext{
* corresponding author; e-mail maryamhosseinpour35@yahoo.com
}

arrangements of air holes in the cladding, which can illustrate the optimal condition for delivery of intensity and also low losses such as confinement loss, while the index of filling liquid is varied.

The importance of study of the output intensity profiles including the central intensity and the width of profiles, appear especially in medical applications such as optical coherence tomography (OCT) imagining [19]. As an example, it is reported that OCT imagining at $1.5 \mu \mathrm{m}$ up to $1.6 \mu \mathrm{m}$ broadband light source can be used to take images of human tooth samples [20].

In our previous work on PCFs, the CL and the group velocity dispersion (GVD) of two types of MOFs against wavelengths were investigated [21]. Moreover, in our early works [22-24], the influence of various configurations and different wavelengths $(1.2 \mu \mathrm{m} \leq \lambda \leq 1.8 \mu \mathrm{m})$ on the intensity distribution, output intensity profiles and confinement loss for the original PCFs without liquid were discussed. We have also investigated the effect of small $d / \Lambda$ values $(d / \Lambda=0.3,0.4)$ on the output intensity profiles [25]. Furthermore, in our recent work [26], optimum values of air filling fraction $(d / \Lambda)$ for photonic crystal fibers with different configurations and fixed number of air rings $(N=4)$ were presented.

In all of our early works, we studied the properties of PCFs having various configurations (starting with square lattice and carried on up to the nanostructure), without any liquids filled in the air-holes. To improve the output intensity of selectively filling air-holes with liquid in PCF structures, the present article investigates the new designs of externally liquid-filled PCFs with four liquidhole layers lying outside the two inner air-hole layers. A Gaussian amp function is applied to evaluate approximately the output intensity profiles of different configurations of the proposed liquid-filled PCFs. By varying the air-hole diameters and liquid indices, the central intensity, full width at half maximum, effective index, and also confinement loss of the considered PCFs are investigated.

As far as we know, all of the previous papers have only been focused on the liquid-filled PCFs with a uniform structural parameters such as the same values of hole dimensions in the cladding region. However, our 
recent achievements illustrate that such design parameters as the number of edges $(E)$ in the cladding and the dimensions of holes are the main factors of concern in the context of high output intensity with small full width at half maximum (FWHM) and low confinement loss. Considering the key roles of liquid filled PCFs for sensing applications, the main focus of this research is thus to present the most promising designs of these fibers which can improve significantly their performances for any sensing applications.

Indeed, the main topic of this work is the investigation of the effect of unique designs to optimize the potential of liquid-filled PCFs. According to our simulations, the presence of large air-hole sizes, particularly in the first ring compared to the smaller liquid-filled holes embedded in the other rings can influence the output intensity distribution remarkably. The emergence of liquid-filled holes in the cladding region can be advantageous due to its remarkable positive effects on the strong confinement of the intensity in the core compared to our previous results obtained for the PCFs without any liquids. Furthermore, the significance of our analysis on the liquid-filled PCFs can be revealed in the field of plasma physics, in which we encounter with plasma waveguides having plasma in the cladding instead of liquid [27]. Additionally, it is possible to add a dopant to the liquids infiltrated into the holes of the PCFs. This can be useful to increase the index of filling liquid, leading to an increment in the output intensity due to the greater refractive index difference between core and cladding regions. From the bend point of view, it is worth noting that obtaining a promising PCF design still represents a technical challenge. Thus, further investigations will have to clarify the practical impact of the holes filled with liquids on the efficiency of these PCFs. As a result, the fiber designs presented in this study for liquid-filled PCFs can be appropriate for both sensing and such medical applications as OCT, which requires to be done with high accuracy. This is a consequence of the fact that the examined structures, especially the externally liquid PCFs with octagonal and nanoconfigurations possess sharp output intensity profiles.

Furthermore, numerical results show the configurations proposed for the externally liquid-filled PCF with octagonal lattice and nanodesign rather than other examined PCFs, not only boost the output intensity noticeably, but also limit the CL to nearly $0 \mathrm{~dB} / \mathrm{m}$ even when the liquid index $n_{q}$ is varied from $n_{q}=1.2$ to $n_{q}=1.6$. In other words, it can be stated that the nanoconfiguration with the unique design presented in this work, appears to be the best qualified for a new class of liquidfilled PCFs, due to its highest output intensity with very low loss.

\section{Results and discussions}

Please note that in the following discussion, the number of rings $(N)$ are fixed on $N=6$, which is found to be the optimum value of this parameter for achieving the remarkable output intensity with very low CL [22].
Moreover, fabricating the designs with $N>6$, especially for PCFs with more number of edges in the cladding (e.g. octagonal or nanostructures) seems to be uneconomical or very difficult. As far as we know, previous studies mainly focused on a hexagonal structure of selectively liquid-filled PCFs with the same values of the $d / \Lambda$ ratio for all of the hole layers. However, in this work, the air-filling fraction value of the first ring is kept constant on $d / \Lambda=0.8$. It is reported that for the externally liquid-filled PCFs, the outer liquid-hole layers can decrease the use of the lossy liquid with the inner air-hole layers surrounding the solid core to maintain the singlemode operation by the totally internal reflection (TIR) guiding [18].

Hence, in the present study, the four outer air-hole layers are respectively filtrated with the high and low index-liquid lying outside the two inner air-hole layers. Furthermore, the $d / \Lambda$ ratio of the first air-hole ring for all of the proposed externally liquid-filled PCFs, is unchanged, whereas diameter of the holes in the five outer rings is decreased while $\Lambda$ remains constant resulting in the lower values of $d / \Lambda$. Additionally, our previous achievements revealed that despite ascending the output intensity, PCFs with the extra number of edges $(E>9)$ in the cladding cannot be useful due to the fact that such structures not only offer the large amounts of CL but also show side dips on the envelope of the intensity profiles [22]. Therefore, the PCF with nanoconfiguration is the final studied externally liquid-filled PCF.

Here, the COMSOL Multiphysics v3.4 software is applied to simulate the PCFs and to obtain the output intensity profiles. All the output profiles obtained in the preceding have been digitized using the XY Extract Graph Digitizer v5.1 software to find the coordinates of the simulated profiles with great accuracy. These obtained dots are considered as digitized data. In the following step, the digitized data of actual profiles are then approximated using the analytical software, Origin Pro v8.6, with Gaussian amp function which is defined by Eq. (1) [28]:

$$
I(x)=I_{0} \exp \left(\frac{-x^{2}}{2 w^{2}}\right),
$$

where $I_{0}$ signifies the central intensity, the parameter $w$ is standard deviation and $x$ is a position of each point in which intensity is calculated for that point (in $\mu \mathrm{m}$ ).

The numerical method we employed to find out the propagation properties of the selectively liquid-filled PCFs is also an efficient and accurate numerical method for the analysis of the original PCF structures without any liquids filled in the air-holes [22-26]. Besides the central intensity, the parameter FWHM which is an important parameter is investigated using the Origin Pro software to estimate the width of output intensity profiles. The values obtained for this parameter are more near to the amounts which are found with the relationship that has been employed by Wolfram Math World [29].

After finding out the effective mode indices $\left(n_{\text {eff }}\right)$ of the PCFs, the confinement loss can then be calculated using the following equation [30]: 
$L_{c}=8.686 k_{0} \operatorname{Im}\left(\mathrm{n}_{\mathrm{eff}}\right)$,

where $L_{c}$ signifies CL, $\operatorname{Im}\left(\mathrm{n}_{\mathrm{eff}}\right)$ is the imaginary part of effective mode index and $k_{0}=2 \pi / \lambda$.

A perfectly matched layer (PML) is implemented for the calculation of the modal CLs. We consider two kinds of externally liquid-filled PCFs with their cross-sections demonstrated in Figs. 1 and 2.



Fig. 1. Cross-sections of the various configurations of externally liquid-filled PCFs with the four outer liquidhole layers lying outside the two inner air-hole layers. Diameter of the air-holes except those located in the first ring is $d_{1}$ and $d$ is the diameter of holes in the first ring.

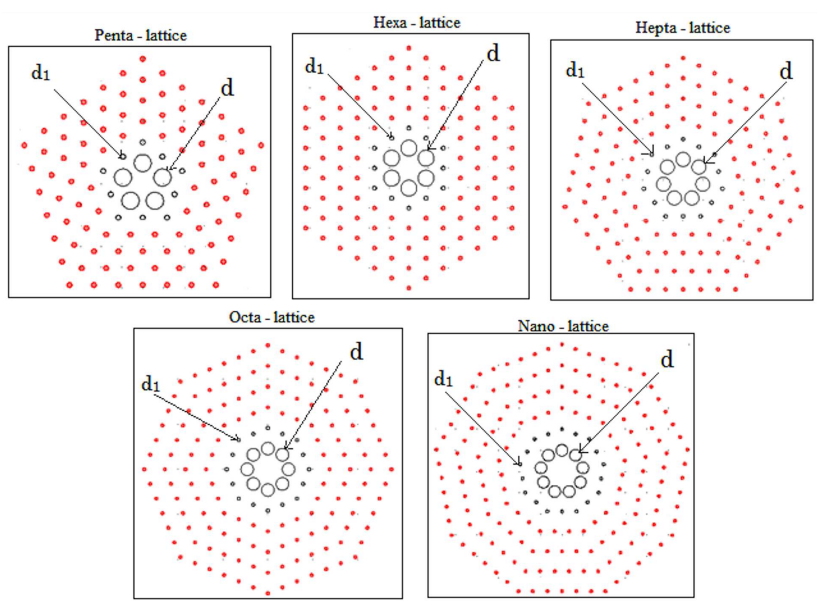

Fig. 2. Cross-sections of the various configurations of externally liquid-filled PCFs with the four outer liquidhole layers lying outside the two inner air-hole layers and with the lower values of $d_{1}$.

By selectively filling the high-index liquid into the outer air-hole layers of the PCFs, we can have externally liquid-filled PCFs with the solid core surrounding by the inner air-hole layers. As mentioned before, the number of rings must be reduced as far as possible in the design phase to facilitate the fabrication process. Hence, the proposed fibers contain six rings in their cladding, the four outer liquid-hole layers lying outside the two inner air-hole layers. All the fiber designs proposed in the following have been simulated according to the building instruction for equilateral polygons based on [31]. The shape of air-holes for all the examined fibers is circular. Additionally, the radius of the second, third, fourth, fifth, and sixth air rings is, respectively, twice, three, four, five, and six times larger than the radius of the first ring which has been supposed equal to $2.3 \mu \mathrm{m}$.

We first consider the externally liquid-filled PCFs with different configurations starting with the pentagonal lattice and carried on up to the nanostructure shown in Fig. 1 with the refractive index of the liquid $n_{q}$ being 1.2 and 1.6, respectively. Moreover, the refractive index of the cladding is $n_{\text {clad }}=1.44$. The diameter of the airholes except those located in the first ring is $d_{1}$, and also the diameter of holes in the first ring is $d$; the lattice constant of the studied structures is $\Lambda$. Additionally, the dimension of holes is chosen in a way that $d / \Lambda$ remains constant when another effective parameter $\Lambda$ decreases as the number of edges in the cladding region $(E)$ rises. The dimension of air holes $\left(d, d_{1}\right)$ and also the lattice constant $(\Lambda)$ for the various PCF configurations are given in Table I.

TABLE I

The structural parameters of the examined PCFs with $d / \Lambda=0.8$ and $d_{1} / \Lambda=0.6$.

\begin{tabular}{c|c|c|c}
\hline \hline Structures & $\Lambda[\mu \mathrm{m}]$ & $\begin{array}{c}d[\mu \mathrm{m}] \\
d / \Lambda=0.8\end{array}$ & $\begin{array}{c}d_{1}[\mu \mathrm{m}] \\
d_{1} / \Lambda=0.6\end{array}$ \\
\hline pentagonal & 2.702 & 2.1616 & 1.6212 \\
hexagonal & 2.3 & 1.84 & 1.38 \\
heptagonal & 1.9972 & 1.5978 & 1.1983 \\
octagonal & 1.761 & 1.4088 & 1.0566 \\
nano & 1.5734 & 1.2587 & 0.944
\end{tabular}

Our previous results revealed that when $d / \Lambda \geq 0.5$, the output intensity is markedly concentrated in the core. As an example, the heptagonal, octagonal and nanodesigns with $d / \Lambda=0.8$ offered the highest output intensity with very low loss. Additionally, for $\lambda=1.55 \mu \mathrm{m}$ and $1.65 \mu \mathrm{m}$, it was shown that $d / \Lambda=0.6$ is suitable for achieving the maximum output intensity with very low confinement loss [26]. Accordingly, the $d / \Lambda$ ratio of the first air-hole ring for all of the proposed externally liquidfilled PCF structures, is fixed on $d / \Lambda=0.8$, whereas diameter of the holes in the five outer rings is decreased, while $\Lambda$ is constant resulting in the lower value of air-filling fraction $d / \Lambda=0.6$.

The existence of the air filled holes in the first two rings, especially those located on the first (inner) ring with larger values of the air-hole diameters $(d)$, results in the greater confinement of the intensity in the core.

To see the shape of the output light in two dimensions, Fig. 3 shows the two-dimensional (2D) intensity distributions of the PCFs considered with four outer liquid-hole layers lying outside two inner. air-hole layers, at $\lambda=1.55 \mu \mathrm{m}$ for $n_{q}=1.2$ which all are obtained using the COMSOL software. At this point, please note 

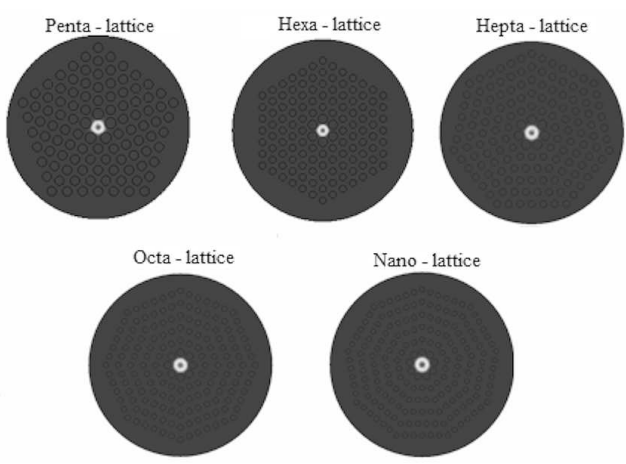

Fig. 3. Simulated 2D intensity distributions related to the various designs of the externally liquid-filled PCFs with $d_{1} / \Lambda=0.6$ for all rings except the first ring and $d / \Lambda=0.8$ for the inner (first) layer, while $N=6, n_{q}=$ 1.2 , and $\lambda=1.55$. Surface of the plots represents the intensity distributions $\left(\mathrm{W} / \mathrm{m}^{2}\right)$ related to the examined fibers.
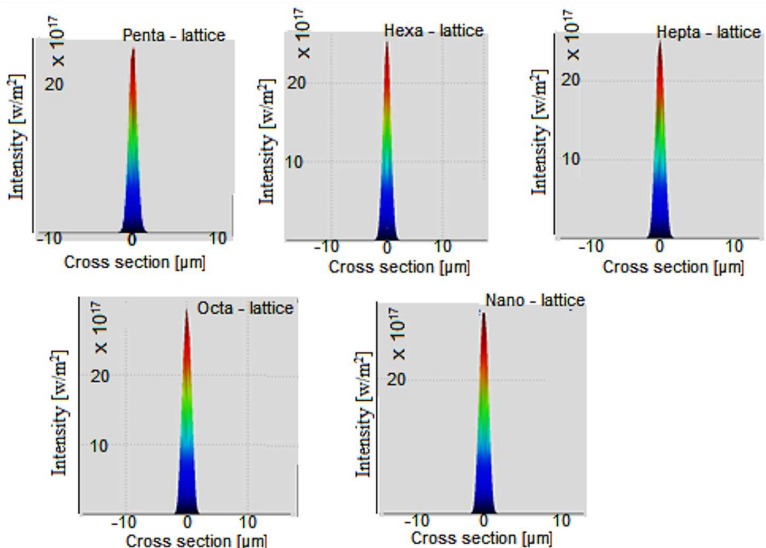

Fig. 4. Simulated output intensity profiles belonging to the various designs of the externally liquid-filled PCFs with $d_{1} / \Lambda=0.6$ for all rings except the first ring and $d_{,} \Lambda=0.8$ for the inner (first) layer, while $N=6, n_{q}=$ 1.2 , and $\lambda=1.55$. The vertical axis represents intensity $\left(\mathrm{W} / \mathrm{m}^{2}\right)$, and the horizontal axis refers to cross-sections of the examined PCFs (in $\mu \mathrm{m}$ ).
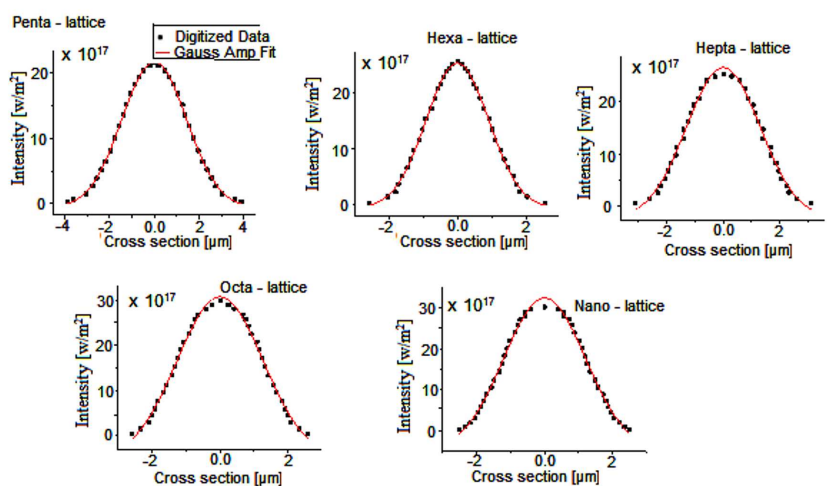

Fig. 5. Approximated output intensity profiles related to the various designs of the externally liquid-filled PCFs with $d_{1} / \Lambda=0.6$ and $d / \Lambda=0.8$, while $N=6, n_{q}=1.2$, and $\lambda=1.55$. The vertical axis represents intensity $\left(\mathrm{W} / \mathrm{m}^{2}\right)$, and the horizontal axis refers to cross-sections of the examined PCFs (in $\mu \mathrm{m}$ ).

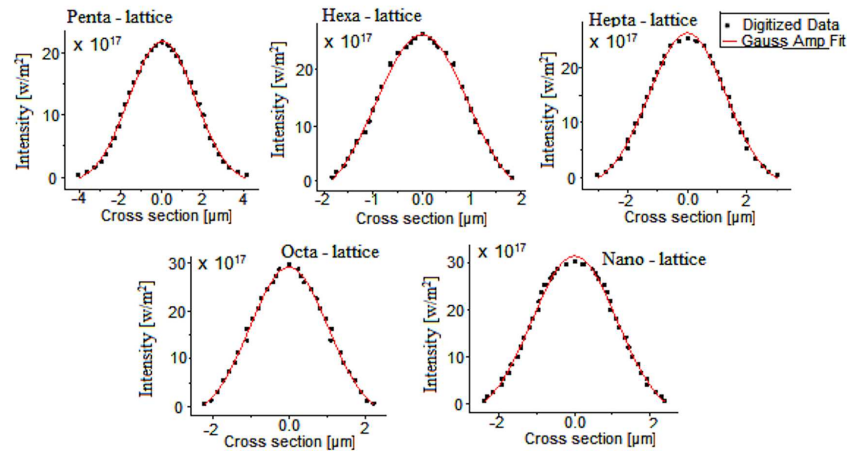

Fig. 6. Approximated output intensity profiles related to the various designs of the externally liquid-filled PCFs with $d_{1} / \Lambda=0.6$ and $d / \Lambda=0.8$, while $N=6, n_{q}=1.6$, and $\lambda=1.55$. The vertical axis represents intensity $\left(\mathrm{W} / \mathrm{m}^{2}\right)$, and the horizontal axis refers to cross-sections of the examined PCFs (in $\mu \mathrm{m}$ ).

that in Fig. 3, surface of the plots represents the intensity distributions $\left(\mathrm{W} / \mathrm{m}^{2}\right)$ related to the examined fibers. The corresponding output intensity profiles are also displayed in Fig. 4.

In Fig. 4, the vertical axis refers to intensity $\left(\mathrm{W} / \mathrm{m}^{2}\right)$, while the horizontal axis represents cross-sections of the examined PCFs (in $\mu \mathrm{m}$ ). These profiles are then analyzed and approximated using the proposed function with high accuracy and are illustrated in Fig. 5 for liquid with lower index $n_{q}=1.2$. Note that these approximated profiles are exactly the results obtained utilizing the analytical software (Origin Pro).

As mentioned before, the COMSOL software is applied to simulate cross-sections of the PCF with different structures, but there is no consideration for the length of the fibers in this study. The approximated output intensity profiles obtained for the liquid with higher index, $n_{q}=1.6$, are also displayed in Fig. 6

Figures 5 and 6 demonstrate that the digitized data (solid dots) of the simulated profiles fit well to the Gaussian function (solid curve) when the number of edges $(E)$ is increased. This approximation is also accurate as the liquid indices is varied from $n_{q}=1.2$ to $n_{q}=1.6$. Please note that in Fig. 5 and Fig. 6 the vertical axis refers to intensity $\left[\mathrm{W} / \mathrm{m}^{2}\right]$ and the horizontal axis represents cross-sections of the examined PCFs (in $\mu \mathrm{m}$ ).
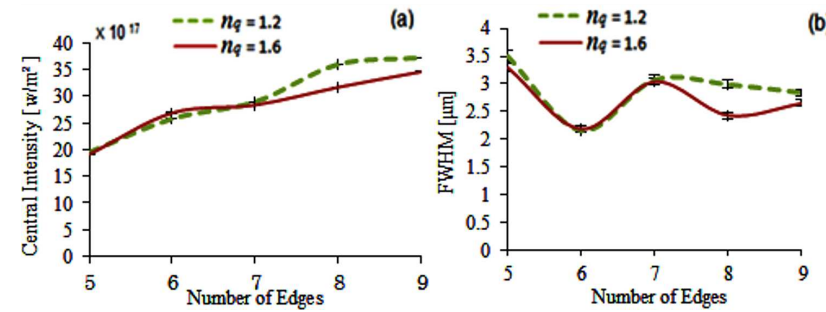

Fig. 7. Plots of (a) central intensity and (b) FWHM against the number of edges in cladding region for various liquid indices $n_{q}$ when $d / \Lambda=0.8$ and $d_{1} / \Lambda=0.6$ and $N=6$ at $\lambda=1.55 \mu \mathrm{m}$. 
Figure $7 \mathrm{a}$ and b summarizes the dependence of the central intensity and the number of edges in the cladding $(E)$ as well as the relationship between the parameter FWHM and $E$, respectively, for $n_{q}=1.2$ and 1.6 , at $\lambda=1.55 \mu \mathrm{m}$ which is the wavelength of choice in most telecom applications. The errors reported by the software which are involved with the approximation are shown as error bars in Fig. 7.

In our early works [22-26], it was obtained without any liquids filled in the air-holes, output intensity of the original PCFs enhances as the number of edges in the cladding rises. As the four outer air-hole layers are filled with liquid, this remarkable increment can still be seen in the corresponding values of the central intensity versus the number of edges as demonstrated in Fig. 7.

One parameter which has a considerable impact on the characteristics of the PCF is the number of the airholes. Moreover, one point that is worth to notice is that increase of the number of edges in the cladding raises the number of holes in each ring. As an example, there are 18 air-holes in the first two rings of the externally liquid-filled PCF considered with nanolattice, whereas, the PCF with pentagonal design has only 10 air-holes in these first two rings (see Fig. 1). Indeed, the refractive index difference between core and cladding region rises as the number of edges increases, because of the emergence of the additional air holes. This leads to the strong confinement of the intensity in the core; hence the central intensity boosts remarkably. It can be seen that the central intensity plots as a function of the parameter $E$ show approximately linear behavior. The slope of central intensity curves remains almost constant with variations of the liquid index; nevertheless these variations affect the central intensity values. As shown in Fig. 7b, variation occurring in the parameter FWHM is different. That is, the FWHM values vary within a small range even when we change the liquid index. The marked decrease in the FWHM plots occurs exactly where the PCF structure is varied from pentagonal lattice to a hexagonal structure. This behavior can be due to the diameter of the holes in the first air-hole layer of the PCF with hexagonal lattice $(d=1.84 \mu \mathrm{m})$ is getting closer to the used wavelength $\lambda=1.55 \mu \mathrm{m}$.

By varying the liquid index from $n_{q}=1.2$ to $n_{q}=1.6$, the PCFs considered with octagonal and nanostructures (the configurations with more number of edges in the cladding), still boost the central intensity considerably and reduce the parameter FWHM. Please note that the dimension of the holes in the PCFs with more number of edges (the PCF with octagonal and nanoconfigurations) are getting closer to the employed wavelength $\lambda=1.55 \mu \mathrm{m}$ than the other considered PCFs. Indeed, the radius of the air-holes $(r=d / 2)$ in the first ring of the PCFs with octagonal and nanodesigns $(r=0.704$ and $0.629 \mu \mathrm{m}$, respectively) are smaller than half of the operating wavelength $(\lambda / 2=0.775 \mu \mathrm{m})$ (see Table I). Accordingly, with increasing the liquid index from $n_{q}=1.2$ to $n_{q}=1.6$, the value of central intensity as well as the
FWHM parameter related to the PCFs with octagonal and nanostructures decreases due to the refractive index of the cladding region is raised. On the contrary, where the PCF configuration is changed from pentagonal lattice to a heptagonal structure, considering the error bars, the values of both the central intensity and the FWHM parameter remain almost constant as we change the liquid index (see Fig. 7).

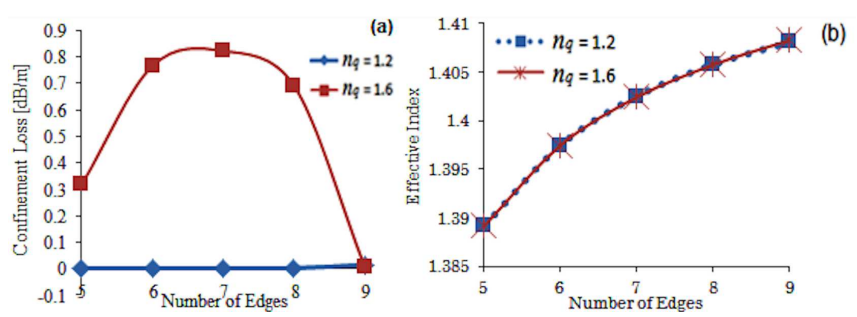

Fig. 8. Plots of (a) confinement loss and (b) effective indices against the number of edges when $d / \Lambda=0.8$ and $d_{1} / \Lambda=0.6, N=6$ at $\lambda=1.55 \mu \mathrm{m}$.

The plots of calculated confinement loss and also the corresponding effective index curves for variations in $n_{q}$ are depicted in Fig. 8a and b, respectively.

The existence of air-hole layers around the center solid core results in the guiding mechanism remaining the TIR effect. Additionally, we can see the externally liquid-filled PCFs with more number of edges have the larger effective index due to the appearance of the additional holes leading to the grater refractive index difference between core and cladding region. In fact, the effective index increases as the number of edges in the cladding rises, yet the slope of the effective index plots remains unchanged when we vary the liquid index from $n_{q}=1.2$ to $n_{q}=1.6$.

Figure $8 \mathrm{a}$ shows that for $n_{q}=1.2$, the value of confinement loss drops considerably in all of the proposed PCFs. It is predicted that this linear behavior is because the liquid with lower index $n_{q}=1.2$ leads to the greater refractive index difference between core and cladding. This results in the minimum of loss (roughly $0 \mathrm{~dB} / \mathrm{m}$ ) obtained for $n_{q}=1.2$. On the other hand, one can see that for liquid with higher index $n_{q}=1.6$, confinement losses of the PCFs with pentagonal design up to octagonal structure are increased.

One point that is worth to notice according to Fig. 8a is that as we further vary the liquid index from $n_{q}=1.2$ to $n_{q}=1.6$, only confinement loss of the PCF with nanoarrangement remains unchanged because of its geometry. Please note that the proposed fiber with nanoconfiguration has the lowest value of lattice constant $\Lambda=1.5734 \mu \mathrm{m}$.

To increase the central intensity further, diameter of the holes in the five outer rings of the considered externally liquid-filled PCFs is modified. Figure 2 shows the cross-sections of the externally liquid-filled PCFs considered in the following discussion.

As shown in Fig. 2, the only difference between the liquid-filled PCFs considered in the earlier stage and 
those proposed in the following is the value of $d_{1}$. Indeed, as can be seen in Fig. 2 and also Table II, we will diminish the values of air-hole diameter in the five outer rings while another design parameter $\Lambda$ is constant resulting in the lower $d_{1} / \Lambda$ ratio $\left(d_{1} / \Lambda=0.2\right)$. However, the dimension of air holes in the first ring $(d)$ is assumed to be unchanged (the $d / \Lambda$ ratio of the inner ring is still fixed on 0.8).

\section{TABLE II}

The structural parameters of the examined PCFs with $d / \Lambda=0.8$ and $d_{1} / \Lambda=0.2$.

\begin{tabular}{c|c|c|c}
\hline \hline Structures & $\Lambda[\mu \mathrm{m}]$ & $\begin{array}{c}d[\mu \mathrm{m}] \\
d / \Lambda=0.8\end{array}$ & $\begin{array}{c}d_{1}[\mu \mathrm{m}] \\
d_{1} / \Lambda=0.2\end{array}$ \\
\hline pentagonal & 2.702 & 2.1616 & 0.5404 \\
hexagonal & 2.3 & 1.84 & 0.46 \\
heptagonal & 1.9972 & 1.5978 & 0.39944 \\
octagonal & 1.761 & 1.4088 & 0.3522 \\
nano & 1.5734 & 1.2587 & 0.31468
\end{tabular}
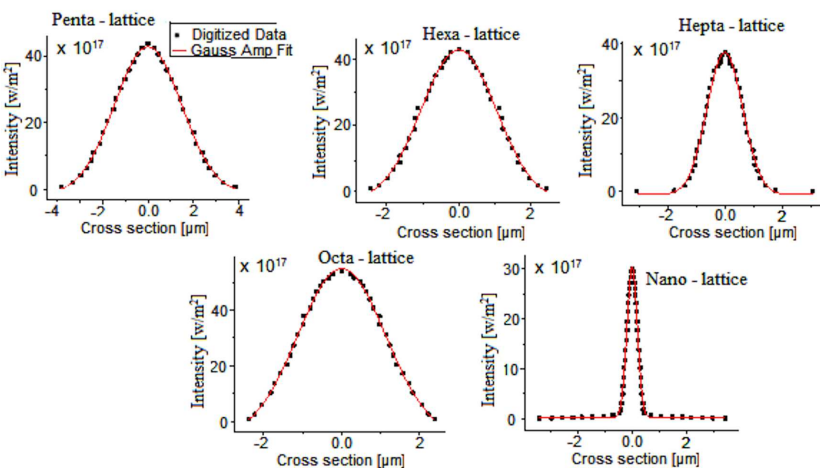

Fig. 9. Approximated output intensity profiles belonging to the various designs of the externally liquid-filled PCFs with $d_{1} / \Lambda=0.2$ and $d / \Lambda=0.8$, while $N=6$, $n_{q}=1.6$, and $\lambda=1.55 \mu \mathrm{m}$. The vertical axis represents intensity $\left(\mathrm{W} / \mathrm{m}^{2}\right)$, and the horizontal axis refers to cross-sections of the examined PCFs (in $\mu \mathrm{m}$ ).

The results obtained for the externally liquid-filled PCFs proposed with lower values of $d_{1}$ are shown in Fig. 9 for $n_{q}=1.6$ at $\lambda=1.55 \mu \mathrm{m}$.
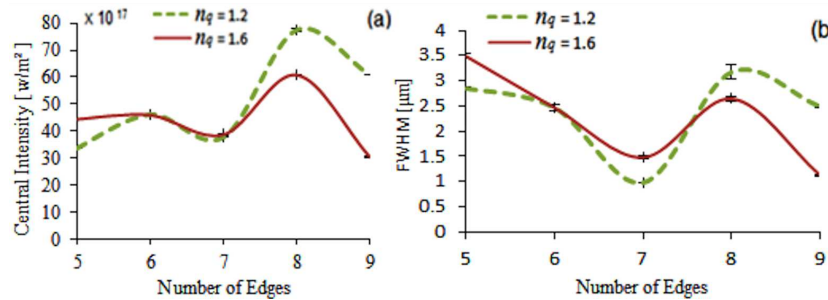

Fig. 10. Plots of (a) central intensity and (b) FWHM against the number of edges in the cladding region for various liquid indices $n_{q}$ when $d / \Lambda=0.84$ and $d_{1} / \Lambda=$ 0.2 , and $N=6$ at $\lambda=1.55 \mu \mathrm{m}$.

Figure $10 \mathrm{a}$ and b summarizes, respectively, the variation between the central intensity and the number of edges in the cladding as well as the variation between the parameters FWHM and $E$ (number of edges) for lower values of $d_{1}$, at $\lambda=1.55 \mu \mathrm{m}$. It is clearly shown in Fig. 10a that variations in the dimension of the five outer rings have great impact on the central intensity values, especially in the liquid-filled PCFs with pentagonal, hexagonal, and octagonal configurations.

As shown in Fig. 10a, the marked increment is still seen in the central intensity plots as a function of $E$. Besides, FWHM shows a calm reduction with $E$ which is in a good agreement with Fig. 9, when a narrower intensity profile (sharper intensity profile) is obtained by increasing the number of edges. Compared to Fig. 7a, one can see that the highest central intensity (nearly $80 \times 10^{17} \mathrm{~W} / \mathrm{m}^{2}$ ) is offered by the octagonal lattice of externally liquid-filled PCF with lower values of $d_{1}$. Indeed, lower values of $d_{1}$ (diameter of holes in the five outer rings) for the externally liquid-filled PCFs with pentagonal, hexagonal, and octagonal designs boost the central intensity values almost twice larger than those of the corresponding structures with higher values of $d_{1}$.

As an example, for $n_{q}=1.2$, when $d_{1}=1.0566 \mu \mathrm{m}$ which results in $d_{1} / \Lambda=0.6$, the PCF with octagonal lattice increases the central intensity to roughly $35.97 \times 10^{17} \mathrm{~W} / \mathrm{m}^{2}$, whereas for $d_{1}=0.3522 \mu \mathrm{m}$ leading to $d_{1} / \Lambda=0.2$, the intensity exceeds $77.44 \times 10^{17} \mathrm{~W} / \mathrm{m}^{2}$. Please note that for small $d / \Lambda$ values $(\leq 0.3)$, the tail of the confined intensity extends into the cladding that results in the emergence of side dips on the envelope of the intensity profile for the original PCFs without liquid [22]. However, by looking at Fig. 9, it is obvious that the presence of holes with both the small and large air-filling fraction values does not only delete these sorts of side dips but also leads to the marked increment in the central intensity. Figure 11a shows the confinement loss curves versus the number of edges $(E)$ for variant values of $n_{q}$. Additionally, the corresponding effective index curves are displayed in Fig. 11b.

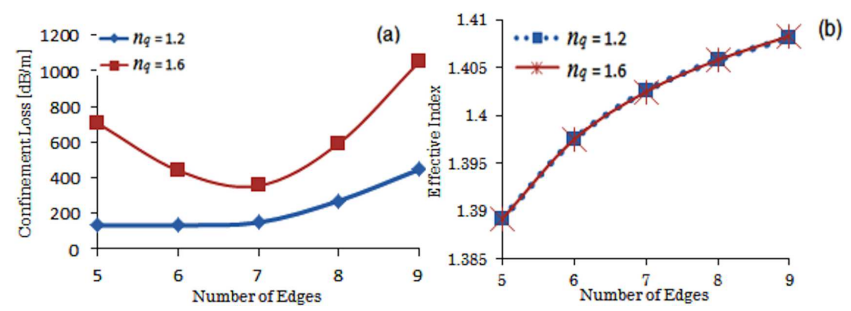

Fig. 11. Plots of (a) confinement loss and (b) effective indices against the number of edges when $d / \Lambda=0.8$ and $d_{1} / \Lambda=0.2, N=6$ and $\lambda=1.55 \mu \mathrm{m}$.

Similar to the externally liquid-filled PCFs considered with larger values of $d_{1}$, the effective indices of our proposed PCFs with lower values of $d_{1}$ increases as the number of edges in the cladding rises. However, Fig. 11a clearly demonstrates that the confinement losses of our proposed PCFs with lower values of $d_{1}$ increase dramatically. As an example, when $n_{q}=1.2$, the pentagonal design with lower values of $d_{1}$ limits CL to $132.21 \mathrm{~dB} / \mathrm{m}$, whereas the 
corresponding structure with higher values of $d_{1}$ can dramatically reduce the loss about $0.125 \times 10^{-5} \mathrm{~dB} / \mathrm{m}$.

Compared with Fig. 8a, one can see that the change in the liquid index drastically shifts confinement loss curve towards larger values. As shown in Fig. 11a, for lower values of $d_{1}$, when $n_{q}=1.2$, by varying the fiber design from pentagonal lattice up to heptagonal structure, confinement loss hardly changes; nevertheless, the PCFs with octagonal and nanoconfigurations increase the loss values exponentially.

By comparing the results in Figs. 8a and 11a, it should be indicated that the increment in the values of $d_{1}$ leads to the greater difference in refractive index between core and cladding region. Thus, the intensity is confined greatly in the core, which results in decrease of the loss.

However, smaller values of $d_{1}$ have less impact on decrease of the confinement loss. Indeed, decreasing $d_{1}$ while keeping $\Lambda$ constant, results in a lower air-filling fraction ratio and the refractive index of the cladding rises; hence the confinement loss increases drastically. To attain optimized designs which exhibit the highest output intensity and also the least confinement loss, the externally liquid filled PCFs considered with larger values of $d_{1}$ can be more suitable than those of the corresponding structures with lower values of $d_{1}$.

From these simulation results, it is obtained that by decreasing the air-hole diameters of the five outer rings except the first air-hole layer, the central intensity can be remarkably increased. Besides, it can be seen that four liquid-hole layers lying outside the two inner airhole layers in the externally liquid-filled PCFs proposed with higher values of $d_{1}$ which results in a larger air-filling fraction, can be more favorable for decreasing the CL (nearly $0 \mathrm{~dB} / \mathrm{m})$. Thus, our proposed externally liquid-filled PCFs possess the potential to be employed for optical devices with high output intensities and also low losses at the desired wavelength $\lambda=1.55 \mu \mathrm{m}$.

\section{Conclusions}

In the present study, a Gaussian amp function is applied for approximating the output intensity profile of two kinds of externally liquid-filled PCFs proposed with different air-hole designs. The only difference in their structures is the value of $d_{1}$. Furthermore, the effective index curves are obtained and compared for both of the structures. With increasing $n_{q}$, the effective index plots show similar behavior for designs with lower and higher values of $d_{1}$. From the confinement loss point of view, the structures considered with larger values of $d_{1}$ can efficiently reduce the loss.

As the air-hole diameters of the five outer rings $\left(d_{1}\right)$ are getting closer to those located on the first ring $(d)$, the externally liquid-filled PCFs, especially with octagonal and nanostructure seem to be the most appropriate, as they exhibit the least confinement loss and also the highest output intensity at the desired wavelength for optical communications $\lambda=1.55 \mu \mathrm{m}$. The value of central intensity and confinement loss for the nanoconfiguration with higher value of $d_{1}$ can reach nearly $30 \times 10^{17} \mathrm{~W} / \mathrm{m}^{2}$ and $0 \mathrm{~dB} / \mathrm{m}$, respectively, even by varying the liquid index from $n_{q}=1.2$ to $n_{q}=1.6$.

\section{References}

[1] H. Demir, S. Ozsoy, Opt. Fiber Technol. 17, 594 (2011).

[2] H. Demir, S. Ozsoy, Int. J. Light Electron Opt. 28, 257 (2012).

[3] S. Olyaee, F. Taghipour, J. Phys. Conf. Ser. IOP Publ. 276, 012080 (2011).

[4] S.M. Nejad, N. Ehteshami, in: Proc. IEEE Conf. on Communication Systems Networks and Digital Signal Processing, IEEE Pub., Newcastle upon Tyne 2010, p. 714 .

[5] M.N. Hoque, A. Sayeem, N. Akter, Austral. J. Basic Appl. Sci. 4, 2274 (2010).

[6] X.B. Zheng, Y.G. Liu, Z. Wang, T.T. Han, C.L. Wei, J.J. Chen, Appl. Phys. Lett. 100, 141104-1 (2012).

[7] Y. Wang, M.W. Yang, D.N. Wang, C.R. Liao, IEEE Photon. Technol. Lett. 23, 1520 (2011).

[8] Y.Y. Huang, Y. Xu, A. Yariv, Appl. Phys. Lett. 85, $5182(2004)$

[9] D. Hu, J. Lim, Y. Cui, K. Milenko, Y. Wang, P. Shum, T. Wolinski, IEEE Photon. J. 4, 1248 (2012).

[10] R. Spittel, D. Hoh, S. Bruckner, A. Schwuchow, K. Schuster, J. Kobelke, H. Bartelt, Proc. Photon. Phononic Propert. Eng. Nanostruct. 7946, 79460Z-1 (2011).

[11] Y. Cui, P.P. Shum, D.J. Juan Hu, G. Wang, G. Humbert, X.Q. Dinh, IEEE Photon. J. 4, 1801 (2012).

[12] T. Larsen, A. Bjarklev, D. Hermann, J. Broeng, Opt. Expr. 11, 2589 (2003).

[13] S. Revathi, A. Chandran, S.R. Inbathini, N.S. Raghavee, in: Proc. Advances in Electrical Engineering (ICAEE), Vellore (India), 2014, IEEE.

[14] S. Liu, W. Gao, H. Li, Y. Dong, H. Zhang, Opt. Laser Technol. 64, 140 (2014).

[15] M. Azhar, G. Wong, W. Chang, N. Joly, P.S. Russellin, in: Proc. Science and Innovations (CLEO_SI), San Jose (CA, USA), 2012, paper CTh4B.5.

[16] T. Zhixin, L. Xuejin, C. Yuzhi, F. Ping, Plasmonics 9, 167 (2014).

[17] C.P. Yu, J.H. Liou, Opt. Expr. 17, 8729 (2009).

[18] C.P. Yu, J.H. Liou, S.S. Huang, H.C. Chang, Opt. Expr. 16, 4443 (2008).

[19] F. Begum, Y. Namihira, The Recent Progress in Optical Fiber Research, Ed. Moh. Yasined, In Tech, Rijeka 2012, p. 230.

[20] J.H. Lee, E.J. Jung, C. Kim, in: Proc. IEEE Conf. on Opto Electronics and Communication (IEEE), Hong Kong, 2009, paper FD3.

[21] A. Zendehnam, M. Mirzaei, R. Solgi, Int. J. Opt. Appl. 2, 34 (2012).

[22] M. Hosseinpour, M.Sc. Thesis, Science Faculty, Arak University, Arak 2013.

[23] M. Hosseinpour, A. Zendehnam, in: Proc. 20th Iranian Conf. on Optics and Photonics (ICOP) \&s the 6th Iranian Conf. on Photonics Engineering and Technology (ICPET), Shiraz (Iran), 2014, p. 513. 
[24] M. Hosseinpour, A. Zendehnam, in: Proc. 20th Iranian Conf. on Optics and Photonics (ICOP) \& the 6th Iranian Conf. on Photonics Engineering and Technology (ICPET), Shiraz (Iran), 2014, p. 517.

[25] A. Zendehnam, M. Hosseinpour, M. Mirzaei, K. Hedayati, J. Sci. Res. 6, 193 (2014).

[26] A. Zendehnam, M. Hosseinpour, M. Mirzaei, K. Hedayati, Appl. Opt. 53, 1075 (2014).

[27] V. Singh, D. Kumar, PIER 89, 167 (2009).
[28] J.T. Verdeyen, Laser Electronics, 3rd ed., Prentice Hall, Englewood Cliffs 1995.

[29] Wolfram Math World, Gaussian Function.

[30] S. Olyaee, F. Taghipour, Inter. Phys. Sci. 6, 4405 (2011).

[31] F. Stutzki, F. Jansen, C. Jauregui, J. Limpert, A. Tünnermann, Opt. Expr. 19, 12081 (2011). 